\section{Factores sociodemográficos y motivacionales asociados a la actividad física en estudiantes universitarios}

\author{
FLORALINDA GARCÍA PUELLO ${ }^{1, a}$, YANETH HERAZO BELTRÁN ${ }^{1, a}$, \\ RAFAEL TUESCA MOLINA ${ }^{2, b}$
}

\section{Levels of physical activity among colombian university students}

\begin{abstract}
Background: Physical inactivity is an important risk factor for chronic diseases in Western societies. Aim: To determine the sociodemographic and motivational factors associated with physical activity in college students. Material and Methods: The International Physical Activity Questionnaire (IPAQ) -short form- and the "motives for physical activities measure-revised" (MPAM-R) questionnaire were applied to 900 university students aged $20 \pm 3$ years (60\% women). The frequency, level and motivations for engaging in physical activity and associated demographic factors were analyzed. Results: Twenty two percent of students were classified as having a high level of physical activity, $54.8 \%$ had a low level and $13.9 \%$ were considered inactive. According to MET consumption, $68.9 \%$ of students are inactive and only $16.8 \%$ of students classified as very active. Ninety two percent of active students performed physical activity for health reasons. A significant association between gender and social motivation was observed. Conclusions: A high prevalence of physical inactivity was found in these students. Males showed higher levels of physical activity than females.

(Rev Med Chile 2015; 143: 1411-1418)
\end{abstract}

Key words: Motivation; Motor activity; Students.

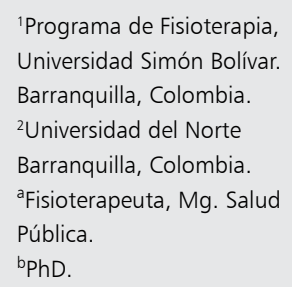

Fuente de apoyo financiero: esta investigación ha sido financiada por la Universidad Simón Bolívar de Barranquilla, la cual, a través del apoyo de la Vicerrectoría de Investigación e Innovación facilitó el proceso de recolección de los datos.

Recibido el 13 de enero de 2015, aceptado el 16 agosto de 2015 .

Correspondencia a:

Floralinda García Puello

Carrera 59 \# 59-65.

fgarcia@unisimonbolivar.edu.co
E xiste suficiente evidencia sobre los beneficios que la actividad física proporciona a la salud, así como también la relación significativa entre niveles bajos de actividad física y enfermedades crónicas no transmisibles (ECNT), las cuales, producen aproximadamente 35 millones de muertes cada año, principalmente en los países de mediano y bajo ingreso ${ }^{1}$. La Organización Mundial de la Salud (OMS) ha declarado que la inactividad física, la dieta inadecuada y el consumo de tabaco y alcohol son los principales responsables de $80 \%$ de estas enfermedades ${ }^{2}$.

Estudios sobre la prevalencia de actividad física en población universitaria muestran datos preocupantes tanto a nivel internacional, como nacional. En Japón, un estudio en universitarios reveló que 46,7\% de hombres y $61,3 \%$ de muje- res no cumplen con las recomendaciones para la práctica de actividad física ${ }^{3}$. En Chile, otro estudio reveló prevalencias de inactividad física entre $89 \%$ y $93 \%{ }^{4}$. En Colombia, un estudio realizado en estudiantes de seis universidades mostró que 40,9\% de los estudiantes pocas veces o nunca hacen algún ejercicio o una práctica corporal por lo menos de 30 min tres veces a la semana, siendo las mujeres las que menos lo realizan ${ }^{5}$.

Los estudiantes universitarios constituyen una población de gran relevancia para el estudio sobre condiciones de salud, en este caso los niveles de actividad física, principalmente por ser un grupo poblacional accesible, homogéneo, que se puede identificar y acceder con facilidad ${ }^{6}$. Además, existe evidencia que las acciones de promoción de la salud de mayor impacto son aquellas orientadas 
a grupos poblacionales cautivos en un contexto institucional, ya sea laboral o educativo. Por lo tanto, las universidades desempeñan un papel protagónico en la promoción de la salud, y su misión debe orientarse no sólo al liderazgo y al desarrollo del conocimiento, sino también a la promoción de estilos de vida saludables en toda la comunidad educativa ${ }^{7}$.

De igual forma, resulta fundamental reconocer que el desarrollo de estrategias de promoción de la salud para el incremento de los niveles de actividad física, requiere del conocimiento de los factores que determinan dicho nivel. Diversos estudios han encontrado que la inactividad física se ha asociado al sexo femenino, mayor edad, nivel socioeconómico bajo y hábito de fumar ${ }^{8}$. Sin embargo, en los últimos tiempos se viene prestando atención a otros posibles determinantes, como son las motivaciones que tienen los individuos para participar en actividades físicas, deportivas y/o recreativas ${ }^{9,10}$.

Las motivaciones son el producto de un conjunto de variables sociales, ambientales e individuales que interactúan determinando la elección de una u otra actividad, la intensidad en la práctica de dicha actividad, la persistencia y el rendimiento. La motivación determina la dirección y la intensidad del esfuerzo, entendiendo esfuerzo como la atracción por ciertas situaciones o actividades, e intensidad como la cantidad de empeño que una persona emplea en una determinada situación ${ }^{11}$. Por tanto, estudios sobre la motivación para la práctica de actividad física resultan fundamentales para conocer porqué algunas personas eligen una determinada actividad y los factores que determinan la permanencia o abandono de esta elección ${ }^{5}$.

Un mejor conocimiento de por qué algunas personas logran mantener una participación en la actividad física, es necesario para orientar el desarrollo de intervenciones de salud pública efectivas que aumenten y mantengan éstos niveles, en este caso en particular de las personas que cursan la universidad. Este estudio tuvo como objetivo la obtención de un diagnóstico sobre los niveles de actividad física de un grupo de estudiantes universitarios y los factores asociados a su práctica, de tal forma que los resultados sirvan como marco de referencia y reflexión sobre las interacciones entre la actividad física, motivaciones y la población universitaria en el diseño de programas de promoción de la actividad física eficaces.

\section{Material y Método}

Se realizó un estudio transversal con una muestra de 900 sujetos. La población a estudiar correspondió a los 8.882 estudiantes pertenecientes a los 15 programas académicos de una institución universitaria en la ciudad de Barranquilla. La muestra fue calculada considerando una prevalencia esperada de actividad física moderada y vigorosa de $30 \%{ }^{12}$, un nivel de confianza de $95 \%$, un error esperado de $3 \%$ y un poder de $80 \%$. Se llevó a cabo un muestreo aleatorio estratificado, y proporcional a la estructura de la población según sexo, edad, programa académico y semestre. Los criterios de selección utilizados fueron: ser mayor de 15 años, estar activo y matriculado en la institución educativa de nivel superior, aceptación voluntaria y firma del consentimiento informado. La aplicación de los instrumentos fue realizada por profesionales entrenados y debidamente formados, entre los meses de septiembre y noviembre de 2013.

Se aplicó un cuestionario de preguntas cerradas para las variables sociodemográficas, el Cuestionario Internacional de Actividad Física (IPAQ) formato corto para la determinación de los niveles de actividad física en población entre los 15 y 69 años de edad, el cual recolecta información sobre la frecuencia, intensidad y duración de la actividad física; los minutos semanales de caminar, de actividad de intensidad moderada y vigorosa. En el presente estudio el alfa de Cronbach fue 0,71, considerándose un nivel aceptable de fiabilidad, el cual coincide con otros estudios $(0,75)^{13}$.

Los datos recolectados mediante el IPAQ formato corto permiten categorizar a los sujetos en distintos niveles. Se considera nivel bajo, sin actividad reportada o no incluido en niveles moderado o alto. Nivel moderado cualquiera de los siguientes: 3 o + días de actividad intensa de al menos 20 min por día, o 5 o más días de actividad moderada y/o caminata de al menos $30 \mathrm{~min}$, o $5 \mathrm{o}$ más días de cualquier combinación de caminata moderada o intensa llegando a 600 METS-minutos por semana. Nivel alto o intenso, es cualquiera de los 2 criterios siguientes: actividad intensa 3 días acumulando 1.500 METS-minuto por semana, 7 o más días de cualquier combinación (caminata, moderada, intensa) acumulando 3.000 METS-minutos por semana. Los niveles de intensidad MET utilizados para la puntuación final fueron: para 
vigorosa $8 \mathrm{MET}$, para moderada 4 MET y para caminar 3,3 MET.

Para la medición de los motivos para la práctica de actividad física se utilizó el cuestionario Motives for Phisycal Activities Measure, revised (MPAM-R). Este instrumento categoriza las motivaciones para la práctica de actividad física en cinco tipos: disfrute, apariencia física, salud, social y competencia.

Los datos fueron analizados mediante el programa estadístico IBM-SPSS ${ }^{\circledR}$ para Windows versión 20 en español, disponible para uso

Tabla 1. Características sociodemográficas de los estudiantes encuestados

\begin{tabular}{|lccc|}
\hline \multicolumn{3}{|c|}{ Frecuencia } & \% \\
Sexo & & & \\
Femenino & 541 & 60,1 & $(56,8 \%-63,3 \%)$ \\
Masculino & 359 & 39,9 & $(36,7 \%-43,2 \%)$ \\
Estado civil & & & \\
Soltero & 838 & 93,1 & $(91,2 \%-94,6 \%)$ \\
Casado & 25 & 2,8 & $(1,8 \%-4,1 \%)$ \\
Unión Libre & 35 & 3,9 & $(2,8 \%-5,4 \%)$ \\
Divorciado & 1 & 0,1 & $(0,0 \%-0,7 \%)$ \\
Separado & 1 & 0,1 & $(0,0 \%-0,7 \%)$ \\
Estrato socioeconómico & & \\
Estrato 1 & 190 & 21,1 & $(18,5 \%-24 \%)$ \\
Estrato 2 & 309 & 34,3 & $(31,2 \%-37,6 \%)$ \\
Estrato 3 & 286 & 31,8 & $(28,8 \%-34,9 \%)$ \\
Estrato 4 & 92 & 10,2 & $(8,4 \%-12,4 \%)$ \\
Estrato 5 & 19 & 2,1 & $(1,3 \%-3,3 \%)$ \\
Estrato 6 & 4 & 0,4 & $(0,1 \%-1,2 \%)$ \\
Edad & Media (DE) & & Rango \\
& $20,1( \pm 3,2)$ años & $15-51$ años \\
\hline
\end{tabular}

institucional. Se estimó la frecuencia del nivel de actividad física, de las motivaciones para la práctica de actividad física y de las variables sociodemográficas. De igual manera se estimó la relación entre los niveles de actividad física, variables sociodemográficas y motivaciones para la actividad física mediante un análisis bivariado que permitió explorar los factores asociados con la práctica de actividad física en los estudiantes, utilizando la prueba $\chi^{2}$, estableciendo el nivel de significación estadística en $\mathrm{p}<0,05$.

Se realizó un análisis de regresión logística con el objetivo de modelar la influencia de las variables sociodemográficas sobre los motivos para la actividad física y de acuerdo a los criterios de Hosmer y Lemeshow, en el modelo se incluyeron las variables que en el análisis de su asociación individual con el evento mostraron un valor $\mathrm{p}<0,25$.

Este estudio fue aprobado por el comité de Ética de la Universidad del Norte, Barranquilla.

\section{Resultados}

Dentro de las principales características sociodemográficas de los estudiantes encuestados se encontró un predominio de mujeres $(60,1 \%)$, solteros $(93,1 \%)$ y de estrato socioeconómico 3 $(34,3 \%)$. La media de edad fue de 20,1 (DE 3,2) años, como lo muestra la Tabla 1.

Con relación a los niveles de actividad física, se observa en la Tabla 2 que 21,6\% se clasifica en nivel alto de actividad física. El mayor porcentaje de los estudiantes $(54,8 \%)$ presentó bajo nivel de actividad física. Tomando como referencia los MET consumidos por semana, se encontró que $68,9 \%$

Tabla 2. Niveles de actividad física en los estudiantes participantes

\begin{tabular}{|lcc|}
\hline & Frecuencia & $\%$ \\
\hline Niveles de actividad física total por semana & & \\
Alto & 194 & $21,6(18,9 \%-24,4 \%)$ \\
Bajo & 493 & $54,8(51,5 \%-58,1 \%)$ \\
Inactiva & 125 & $13,9(11,7 \%-16,4 \%)$ \\
Medio & 88 & $9,8(8 \%-12 \%)$ \\
\hline Niveles de actividad de actividad física según los MET minutos por semana & 620 & $68,9(65,7 \%-71,9 \%)$ \\
$\quad$ Inactivo & 129 & $14,3(12,1 \%-16,8 \%)$ \\
Regularmente activo & 151 & $16,8(14,4 \%-19,4 \%)$ \\
Muy activo & 900 & 100,0 \\
\hline Total & 900 \\
\hline
\end{tabular}


de los estudiantes son inactivos, como lo muestra la Tabla 2. Así mismo, en la Tabla 3 se evidencia mayor proporción de estudiantes masculinos regularmente activos y muy activos tomando como referencia el consumo de METS y los minutos de actividad física vigorosa y moderada realizados durante la semana.

En cuanto a los motivos para realizar actividad física en los estudiantes catalogados como activos, una mayor proporción de estudiantes (92,2\%) realiza actividad física por salud; sigue en orden de importancia la diversión con $84,8 \%$ y competencia con $82,6 \%$ (Tabla 4).

Al relacionar los niveles de actividad física y las características sociodemográficas, se encontró que de $31 \%$ de la población que cumple con las recomendaciones de actividad física son mujeres entre los 26 y 51 años, como se observa en la Tabla 5.

Tabla 3. Consumo de MET de acuerdo al tipo de actividad física realizada

\begin{tabular}{|lccc|}
\hline Actividad física & \multicolumn{1}{c}{$\begin{array}{c}\text { Total } \\
\text { Media (DE) }\end{array}$} & $\begin{array}{c}\text { Mujeres } \\
\text { Media (DE) }\end{array}$ & $\begin{array}{c}\text { Hombres } \\
\text { Media (DE) }\end{array}$ \\
\hline Actividades vigorosas & $614,5( \pm 1.329) \mathrm{MET}$ & $535,8( \pm 51,2) \mathrm{MET}$ & $733,2( \pm 79,5) \mathrm{MET}$ \\
\hline Actividades moderadas & $201,4 \quad( \pm 485) \mathrm{MET}$ & $188,6( \pm 20,1) \mathrm{MET}$ & $220,9( \pm 26,9) \mathrm{MET}$ \\
\hline Caminar & $319,5 \quad( \pm 517) \mathrm{MET}$ & $311,3( \pm 21,7) \mathrm{MET}$ & $331,8( \pm 28,1) \mathrm{MET}$ \\
\hline Total consumo/semana & $1.114,1( \pm 1.717) \mathrm{MET}$ & $1.027 \quad( \pm 71,1) \mathrm{MET}$ & $1.247 \quad( \pm 95) \mathrm{MET}$ \\
\hline
\end{tabular}

Tabla 4. Motivos para realizar actividad física en los estudiantes activos

\begin{tabular}{|lcccc|}
\hline Motivaciones & \multicolumn{2}{c}{ De acuerdo } & \multicolumn{2}{c|}{ Desacuerdo } \\
\hline Diversión & Frecuencia & Frecuencia & 43 & $15,2(11,3 \%-20 \%)$ \\
\hline Competencia & 239 & $84,8(80 \%-88,7 \%)$ & 49 & $17,4(13,1 \%-22,3 \%)$ \\
Apariencia & 233 & $82,6(77,7 \%-86,9 \%)$ & 60 & $21,3(16,6 \%-26,5 \%)$ \\
Salud & 222 & $78,7(73,5 \%-83,4 \%)$ & 22 & $7,8(5 \%-11,6 \%)$ \\
Social & 260 & $92,2(88,4 \%-95 \%)$ & 140 & $49,6(43,7 \%-55,6 \%)$ \\
\hline
\end{tabular}

Tabla 5. Niveles de actividad física según variables sociodemográficas

\begin{tabular}{|c|c|c|c|c|c|}
\hline \multirow{3}{*}{ Características } & \multicolumn{4}{|c|}{ Actividad física } & \multirow{3}{*}{ Valor $\mathbf{p}$} \\
\hline & \multicolumn{2}{|c|}{$\begin{array}{l}\text { Cumple las recomendaciones } \\
\qquad(\mathrm{n}=\mathbf{2 8 2})\end{array}$} & \multicolumn{2}{|c|}{$\begin{array}{l}\text { No cumple las recomendaciones } \\
\qquad(n=618)\end{array}$} & \\
\hline & Frecuencia & $\%$ & Frecuencia & $\%$ & \\
\hline \multicolumn{6}{|l|}{ Sexo } \\
\hline Femenino & 161 & 57,1 & 380 & 61,4 & 0,21 \\
\hline Masculino & 121 & 42,9 & 238 & 38,5 & \\
\hline \multicolumn{6}{|l|}{ Rango de edad } \\
\hline $15-25$ & 107 & 37,9 & 209 & 33,8 & 0,22 \\
\hline $26-51$ & 175 & 62,0 & 409 & 66,1 & \\
\hline \multicolumn{6}{|c|}{ Estrato socioeconómico } \\
\hline $1-2$ & 148 & 52,4 & 351 & 56,7 & 0,22 \\
\hline $3-6$ & 134 & 47,5 & 267 & 43,2 & \\
\hline \multicolumn{6}{|l|}{ Estado civil } \\
\hline Con pareja & 18 & 6,3 & 42 & 6,7 & 0,81 \\
\hline Sin pareja & 264 & 93,6 & 576 & 93,2 & \\
\hline
\end{tabular}


Tabla 6. Relación entre motivaciones para la práctica de actividad física y variables sociodemográficas

\begin{tabular}{|c|c|c|c|c|c|c|c|c|c|c|}
\hline & \multicolumn{2}{|c|}{ Diversión } & \multicolumn{2}{|c|}{ Competencia } & \multicolumn{2}{|c|}{ Apariencia } & \multicolumn{2}{|c|}{ Salud } & \multicolumn{2}{|c|}{ Social } \\
\hline & $\begin{array}{c}\text { De } \\
\text { acuerdo }\end{array}$ & $\begin{array}{l}\text { Desa- } \\
\text { cuerdo }\end{array}$ & $\begin{array}{c}\text { De } \\
\text { acuerdo }\end{array}$ & $\begin{array}{l}\text { Desa- } \\
\text { cuerdo }\end{array}$ & $\begin{array}{c}\text { De } \\
\text { acuerdo }\end{array}$ & $\begin{array}{l}\text { Desa- } \\
\text { cuerdo }\end{array}$ & $\begin{array}{c}\text { De } \\
\text { acuerdo }\end{array}$ & $\begin{array}{l}\text { Desa- } \\
\text { cuerdo }\end{array}$ & $\begin{array}{c}\text { De } \\
\text { acuerdo }\end{array}$ & $\begin{array}{l}\text { Desa- } \\
\text { cuerdo }\end{array}$ \\
\hline \multicolumn{11}{|l|}{ Sexo } \\
\hline Femenino & $\begin{array}{c}133 \\
(55,6 \%)\end{array}$ & $\begin{array}{c}28 \\
(65,1)\end{array}$ & $\begin{array}{c}133 \\
(57,1)\end{array}$ & $\begin{array}{c}28 \\
(57,1)\end{array}$ & $\begin{array}{c}132 \\
(59,5)\end{array}$ & $\begin{array}{c}29 \\
(48,3)\end{array}$ & $\begin{array}{c}150 \\
(57,7)\end{array}$ & $\begin{array}{c}11 \\
(50)\end{array}$ & $\begin{array}{c}70 \\
(49,3)\end{array}$ & $\begin{array}{c}91 \\
(65)\end{array}$ \\
\hline Masculino & $\begin{array}{c}106 \\
(44,4 \%)\end{array}$ & $\begin{array}{c}15 \\
(34,9 \%)\end{array}$ & $\begin{array}{c}100 \\
(42,9)\end{array}$ & $\begin{array}{c}21 \\
(42,9)\end{array}$ & $\begin{array}{c}90 \\
(40,5\end{array}$ & $\begin{array}{c}31 \\
(51,7)\end{array}$ & $\begin{array}{c}110 \\
(42,3)\end{array}$ & $\begin{array}{l}11 \\
(50)\end{array}$ & $\begin{array}{c}72 \\
(50,7)\end{array}$ & $\begin{array}{c}49 \\
(35)\end{array}$ \\
\hline Valor de $p$ & \multicolumn{2}{|c|}{0,24} & \multicolumn{2}{|c|}{0,99} & \multicolumn{2}{|c|}{0,12} & \multicolumn{2}{|c|}{0,48} & \multicolumn{2}{|c|}{$0,008^{*}$} \\
\hline \multicolumn{11}{|l|}{ Edad } \\
\hline $15-25$ & $\begin{array}{c}87 \\
(36,4)\end{array}$ & $\begin{array}{c}20 \\
(46,5)\end{array}$ & $\begin{array}{c}88 \\
(37,8)\end{array}$ & $\begin{array}{c}19 \\
(38,8)\end{array}$ & $\begin{array}{c}87 \\
(39,2)\end{array}$ & $\begin{array}{c}20 \\
(33,3)\end{array}$ & $\begin{array}{c}97 \\
(37,3)\end{array}$ & $\begin{array}{c}10 \\
(45,5)\end{array}$ & $\begin{array}{c}55 \\
(38,7)\end{array}$ & $\begin{array}{c}52 \\
(37,1)\end{array}$ \\
\hline $26-51$ & $\begin{array}{c}152 \\
(63,6)\end{array}$ & $\begin{array}{c}23 \\
(53,5)\end{array}$ & $\begin{array}{c}145 \\
(62,2)\end{array}$ & $\begin{array}{c}30 \\
(61,2)\end{array}$ & $\begin{array}{c}135 \\
(60,8)\end{array}$ & $\begin{array}{c}40 \\
(66,7)\end{array}$ & $\begin{array}{c}163 \\
(62,7)\end{array}$ & $\begin{array}{c}12 \\
(54,5)\end{array}$ & $\begin{array}{c}87 \\
(61,3)\end{array}$ & $\begin{array}{c}88 \\
(62,9)\end{array}$ \\
\hline Valor de $p$ & \multicolumn{2}{|c|}{0,20} & \multicolumn{2}{|c|}{0,89} & \multicolumn{2}{|c|}{0,40} & \multicolumn{2}{|c|}{0,45} & \multicolumn{2}{|c|}{0,78} \\
\hline \multicolumn{11}{|l|}{ Estado civil } \\
\hline Casado/unión libre & $\begin{array}{c}16 \\
(6,7)\end{array}$ & $\begin{array}{c}2 \\
(4,7)\end{array}$ & $\begin{array}{c}13 \\
(5,6)\end{array}$ & $\begin{array}{c}5 \\
(10,2)\end{array}$ & $\begin{array}{c}13 \\
(5,9)\end{array}$ & $\begin{array}{c}5 \\
(8,3)\end{array}$ & $\begin{array}{c}15 \\
(5,8)\end{array}$ & $\begin{array}{c}3 \\
(13,6)\end{array}$ & $\begin{array}{c}9 \\
(6,3)\end{array}$ & $\begin{array}{c}9 \\
(6,4)\end{array}$ \\
\hline $\begin{array}{l}\text { Separado/ } \\
\text { divorciado/soltero }\end{array}$ & $\begin{array}{c}223 \\
(93,3)\end{array}$ & $\begin{array}{c}41 \\
(95,3)\end{array}$ & $\begin{array}{c}220 \\
(94,4)\end{array}$ & $\begin{array}{c}44 \\
(89,8)\end{array}$ & $\begin{array}{c}209 \\
(94,1)\end{array}$ & $\begin{array}{c}55 \\
(91,7)\end{array}$ & $\begin{array}{c}245 \\
(94,2)\end{array}$ & $\begin{array}{c}19 \\
(86,4)\end{array}$ & $\begin{array}{c}133 \\
(93,7)\end{array}$ & $\begin{array}{c}131 \\
(93,6)\end{array}$ \\
\hline Valor de $p$ & \multicolumn{2}{|c|}{0,61} & \multicolumn{2}{|c|}{0,22} & \multicolumn{2}{|c|}{0,48} & \multicolumn{2}{|c|}{0,14} & \multicolumn{2}{|c|}{0,97} \\
\hline \multicolumn{11}{|c|}{ Estrato socioeconómico } \\
\hline Estratos 1-2 & $\begin{array}{l}122 \\
(51)\end{array}$ & $\begin{array}{c}26 \\
(60,5)\end{array}$ & $\begin{array}{c}120 \\
(51,5)\end{array}$ & $\begin{array}{c}28 \\
(57,1)\end{array}$ & $\begin{array}{c}118 \\
(53,2)\end{array}$ & $\begin{array}{c}30 \\
(50)\end{array}$ & $\begin{array}{c}133 \\
(51,2)\end{array}$ & $\begin{array}{c}15 \\
(68,2)\end{array}$ & $\begin{array}{c}74 \\
(52,1)\end{array}$ & $\begin{array}{c}74 \\
(52,9)\end{array}$ \\
\hline Estratos 3-6 & $\begin{array}{l}117 \\
(49)\end{array}$ & $\begin{array}{c}17 \\
(39,5)\end{array}$ & $\begin{array}{c}113 \\
(48,5)\end{array}$ & $\begin{array}{c}21 \\
(42,9)\end{array}$ & $\begin{array}{c}104 \\
(46,8)\end{array}$ & $\begin{array}{c}30 \\
(50)\end{array}$ & $\begin{array}{c}127 \\
(48,8)\end{array}$ & $\begin{array}{c}7 \\
(31,8)\end{array}$ & $\begin{array}{c}68 \\
(47,9)\end{array}$ & $\begin{array}{c}66 \\
(47,1)\end{array}$ \\
\hline Valor de $p$ & \multicolumn{2}{|c|}{0,25} & \multicolumn{2}{|c|}{0,47} & \multicolumn{2}{|c|}{0,66} & \multicolumn{2}{|c|}{0,12} & \multicolumn{2}{|c|}{0,90} \\
\hline
\end{tabular}

Tabla 7. Fuerza de asociación entre variables sociodemográficas y motivos para la actividad física

\begin{tabular}{|lcccc|}
\hline Variables & $\begin{array}{c}\text { Diversión } \\
\text { OR (IC 95\%) }\end{array}$ & $\begin{array}{c}\text { Apariencia } \\
\text { OR (IC 95\%) }\end{array}$ & $\begin{array}{c}\text { Salud } \\
\text { OR (IC 95\%) }\end{array}$ & $\begin{array}{c}\text { Social } \\
\text { OR (IC 95\%) }\end{array}$ \\
\hline Sexo & $0,6(0,3-1,2)$ & $1,6 \quad(0,9-2,7)$ & - & $0,5 \quad(0,3-0,8)^{*}$ \\
Rango de edad & $0,6(0,3-1,1)$ & - & - & - \\
Estado civil & $1,6(0,4-7,8)$ & - & - & - \\
Estrato socioeconómico & $0,7 \quad(0,3-1,3)$ & - & $0,4(0,2-1,2)$ & \\
\hline
\end{tabular}

Se encontró una asociación significativa entre género y motivación social $(\mathrm{p}=0,008)$, evidenciándose que los hombres activos se motivan más por el componente social en comparación con las mujeres, resultados evidenciados en la Tabla 6.

La Tabla 7 presenta la fuerza de asociación entre variables sociodemográficas y motivos para la actividad física, siendo ésta significativa para género y motivación social.

\section{Discusión}

Los resultados obtenidos en este estudio evidencian alta prevalencia de niveles bajos de actividad física en los estudiantes, dato que coincide con el informe de la OMS donde se identifica que $31 \%$ de la población del mundo no realiza ninguna actividad física, ocupando ésta el cuarto lugar entre los factores de riesgo de todas las defunciones a 
escala mundial ${ }^{14}$. Así mismo, estudios realizados a nivel nacional e internacional evidencian que una gran proporción de personas pocas veces o nunca hacen algún tipo de ejercicio o actividad física por lo menos 30 min tres veces a la semana ${ }^{5,15-17}$.

Los resultados obtenidos en términos de mejores niveles de actividad física en los hombres de acuerdo con los minutos por semana, así como también el gasto energético en términos MET, son comparables con un estudio realizado en España donde se encontraron diferencias por sexo al comparar el tiempo semanal dedicado a la actividad física, siendo mayores para el masculino ${ }^{18}$. Así mismo, en Chile se midieron los niveles de actividad física en 4 universidades, encontrando que los estudiantes realizaban actividad física 2 o más veces por semana, siendo los del grupo masculino los más activos ${ }^{6}$. Un estudio realizado en estudiantes universitarios en Cartagena, Colombia, también arrojó diferencias de género en cuanto a los niveles de actividad física, donde las mujeres presentan un menor gasto energético que los hombres ${ }^{19}$.

En general, la mayor prevalencia de estudiantes de género masculino regularmente activos y muy activos tomando como referencia el consumo de METS y los minutos de actividad física vigorosa y moderada semanal es consistente con otros estudios $^{20-23}$.

En relación a los motivos para la práctica de actividad física y su asociación con las variables sociodemográficas, se observa que los hombres activos físicamente se motivan más por el aspecto social en comparación con las mujeres. Este resultado se relaciona con un estudio realizado en España, donde se evidencia que el sexo masculino prefiere en mayor proporción participar en actividades físico deportivas colectivas en comparación con las mujeres que prefieren actividades individuales. Este autor demostró con diferencias significativas que los hombres prefieren participar de deportes como el fútbol, baloncesto y atletismo, mientras que las mujeres prefieren fitness o natación. Asimismo, este estudio mostró que 69,7\% de los hombres practicaban sus actividades físico deportivas con los amigos, mientras que $54,3 \%$ de las mujeres no seguía esta opción ${ }^{24}$.

De igual forma, con relación a las motivaciones que tienen los estudiantes activos para la práctica de actividad física, se encontró que la salud y la diversión son los más importantes, los cuales se relacionan directamente con la tendencia a mantener un estilo de vida saludable. Estos resultados son similares a los arrojados por otros estudios ${ }^{25}$. Por otro lado, los motivos que menos inspiran a los estudiantes activos a realizar actividad física son el mantenimiento de la apariencia física y la competencia, resultado que se asemeja con otro estudio donde las razones o motivos relacionadas con la competencia y lo que el autor llama la estética obtuvieron resultados mucho más bajos que los anteriores ${ }^{26}$.

En esta misma línea, un estudio realizado en España, evidenció que los principales motivos de participación en actividades físico deportivas se relacionan con la salud, la diversión y el mantenerse en forma, principalmente (más de $95 \%$ de las personas consideran estas razones importantes o muy importantes) ${ }^{27}$. En Colombia, la principal motivación que tienen los universitarios para la práctica de actividad física es la salud con un porcentaje de $45,8 \%{ }^{5}$.

Por último, se destaca el resultado significativo obtenido entre sexo y motivo social, lo cual se asocia con otros estudios ${ }^{25,28}$ principalmente los que han aprovechado las redes comunitarias como instrumentos viables en la promoción de políticas relacionadas con la promoción de actividad física. Estos estudios, destacan el papel trascendental que tiene el contacto personal y la relación con los otros para la práctica de actividad física, mientras que se ha comprobado que si estos aspectos no existen estos programas conducen a resultados no satisfactorios. Por lo tanto, se considera que las redes y/o asociaciones comunitarias constituyen un instrumento prometedor para la aplicación de programas de promoción de la actividad física ${ }^{29}$.

Se concluye que el conocimiento sobre los motivos que tienen las personas -este caso en particular los estudiantes universitarios- para practicar cualquier tipo de actividad física, ya sea vigorosa o moderada, facilitará la adaptación de la oferta de programas de actividad física y de sus características a la demanda de acuerdo con las necesidades que la población presenta.

Asimismo, la definición adecuada de patrones de actividad física en individuos que pertenecen a una determinada población de referencia, como en este caso el ámbito universitario, es fundamental en la Epidemiología y Salud Pública, considerando las evidencias de asociaciones entre determinados patrones y factores sociodemográficos, de com- 
portamiento y de salud. Se concluye por tanto, la necesidad de plantear este tipo de estudio con una muestra mayor en la que puedan encontrarse resultados más significativos.

Los resultados de este estudio se convierten en la línea base de actividad física de la institución educativa, posibilitando acciones desde la promoción de la salud que favorezcan entornos y sujetos saludables.

En el presente estudio no fue posible establecer relaciones de causalidad entre las variables observadas debido a la naturaleza transversal del diseño. Otras de las limitaciones de esta investigación es el uso del instrumento IPAQ formato corto, el cual no permitió evaluar los niveles de actividad física en los diferentes dominios (hogar, transporte, trabajo y tiempo libre) sólo la actividad física general; por tanto, se recomienda en próximos estudios aplicar el cuestionario largo del IPAQ.

Agradecimientos: El equipo de investigadores agradece a las Instituciones de Educación Superior que apoyaron el desarrollo del proyecto, especialmente a todos los estudiantes que aceptaron participar en el estudio, y diligenciaron la encuesta.

\section{Referencias}

1. Mendoza D, Urbina A. Actividad física en el tiempo libre y autopercepción del estado de salud en Colombia. Apunts Med Esport 2013; 48 (177): 3-9.

2. Organización Mundial de la Salud. Estadísticas sanitarias mundiales, Suiza, 2012.

3. Okazaki K, Okano S, Hagab S, Seki A, Suzuki H, Takahashi K. One-year outcome of an interactive internet-based physical activity intervention among university students. International journal of medical informatics. 2014; 83 (5): 354-60.

4. Durán S, Castillo M, Vio F. Diferencias en la calidad de vida de estudiantes universitarios de diferente año de ingreso del Campus Antumapu. Rev Chil Nutr 2009; 36 (3): 200-9.

5. Varela M, Duarte C, Salazar I, Lema 1, Tamayo J. Actividad física y sedentarismo en jóvenes universitarios de Colombia: prácticas, motivos y recursos para realizarlas. Colomb. Med 2011; 42 (3): 269-77.

6. Mollinedo F, Ortiz P, Araujo R, Lugo L. Índice de masa corporal, frecuencia y motivos de estudiantes universitarios para realizar actividad física. Educ Med Super 2013; 27 (3): 189-99.
7. Rodríguez F, Palma X, Romo A, Escobar D, Aragú B, Espinoza L, et al. Hábitos alimentarios, actividad física y nivel socioeconómico en estudiantes universitarios de Chile. Nutr Hosp 2013; 28 (2): 447-55.

8. Bauman A, Reis R, Sallis J, Wells J, Loos R, Martin B. Correlates of physical activity: why are some people physically active and others not? Lancet 2012; 380 (9838): 258-71.

9. Yang X, Telama R, Hirvensalo M, Hintsa T, Pulkki Råback L, Hintsanen M, et al. Leadership component of type a behavior predicts physical activity in early midlife. Int J Behav. Med 2012; 19 (1): 48-55.

10. Gregory W, Wilkerson G, Oglesby B. Physical activity promotion in a university community. Using the guide to community preventive services. Acsm's Health \& Fitness journal. 2010; 14 (5): 7-11.

11. Granero A, Gómez M, Abraldes J, Rodríguez SN. Motivos de práctica en el ámbito de la actividad física no competitiva. Espiral. Cuadernos del profesorado. 2011; 4 (7): 15-22.

12. Instituto Colombiano de Bienestar Familiar. Encuesta Nacional de la Situación Nutricional de Colombia, 2010. Bogotá; 2011.

13. León PJ, Fuentes I, González JJ, Fernández A, Costa E, Ramos AM. Actividad física y salud percibida en un sector de la población sevillana; estudio piloto. Rev. int. med. cienc. act. fís. deporte 2011; 10 (41): 164-80.

14. World Health Organization. Global status report on noncommunicable diseases, 2010. Geneva, 2011.

15. Sanabria H, Tarqui C, Portugal W, Pereyra H, Mamani L. Nivel de actividad física en los trabajadores de una Dirección Regional de Salud de Lima, Perú. Rev Salud Pública 2014; 16 (1): 53-62.

16. Sit C, Kerr J, Wong I. Motives for and barriers to physical activity participation in middle-aged Chinese women. Psychol Sport Exerc 2008; 9 (3): 266-83.

17. Roldán E, Lopera M, Londoño F, Cardeño J, Zapata S. Análisis descriptivo de las variables: nivel de actividad física, depresión y riesgos cardiovasculares en empleados y docentes de una institución universitaria en Medellín (Colombia). Apunts. Medicina de l'Esport 2008; 43 (158): 55-61.

18. Camargo L. Orozco J, Hernández Y, Niño GI. Dolor de espalda crónico y actividad física en estudiantes universitarios de áreas de la salud. Rev. Soc. Esp. dolor 2009; 16 (8): 429-36.

19. Herazo Y, Hernández J, Domínguez R. Etapas de cambio y niveles de actividad física en estudiantes universitarios de Cartagena. Salud Uninorte 2012; 28 (2): 298-307.

20. Hallal P, Andersen L, Bull F, Guthold R, Haskell W, 
Ekelund U. Global physical activity levels: surveillance progress, pitfalls, and prospects. Lancet 2012; 380: 247 57.

21. Ozdol Y, Kamil M, Pinar S, Cetin E. Investigation of physical activity levels by gender and residential areas: a case study on students in Akdeniz University. Procedia - Social and Behavioral Sciences 2012; 46: 1581-6.

22. Lee E. Age and gender differences in physical activity and health indicators among Korean adolescents. Journal of Science and Medicine in Sport 2012; 15 (1): 276-7.

23. Spriggs A, Brakta F, Al-Dahir S, Johnson C. Gender differences in physical activity in adolescence and early adulthood: The experience of egyptian youth. Journal of Adolescent Health 2012; 50 (2): 36.

24. Pavón L, Moreno J. Actitud de los universitarios ante la práctica físico deportiva: diferencias por género. Revista de Psicología del Deporte. 2008; 17 (1): 7-23.
25. Aaltonen S, Rottensteiner M, Kaprio J, Kujala UM. Motives for physical activity among active and inactive persons in their mid-30s. Scand J Med Sci Sports 2013; doi: 10.1111/sms.12040.

26. Ruiz F, García M, Díaz A. Análisis de las motivaciones de práctica de actividad física y de abandono deportivo en la Ciudad de La Habana (Cuba). Anales de psicología 2007; 23 (1): 152-66.

27. Alvariñas M, Fernández M, López C. Actividad física y percepciones sobre deporte y género. Revista de Investigación en Educación 2009; 6: 113-22.

28. Lucidarme S, Marlier M, Cardong G, De Bourdeaudhuij I, Willem A. Critical success factors for physical activity promotion through community partnerships. Int J Public Health 2014; 59 (1): 51-60.

29. Reynolds R, McKenzie S, Allender S, Brown K, Foulkes $\mathrm{CH}$. Systematic review of incidental physical activity community interventions. Prev Med 2014; 67: 46-64. 